\title{
Activation of quiescent polypoidal choroidal vasculopathy after membrane peeling vitrectomy for epiretinal membrane: a case report
}

\author{
Yuelin Wang ${ }^{1,2+}$, Zhe Chen ${ }^{1,2+}$, Weihong $Y u^{1,2}$ and Youxin Chen ${ }^{1,2^{*}}$
}

\begin{abstract}
Background: Regular membrane peeling vitrectomy for epiretinal membrane (ERM) patients seldom causes large pigment epithelial detachment (PED). We presented an unusual case of the activation of quiescent polypoidal choroidal vasculopathy (PCV) after membrane peeling vitrectomy for ERM, with an uneven therapeutic process.

Case presentation: A 75-year-old female patient complained of metamorphopsia in her left eye for 2 years. Her best-corrected visual acuity was 20/160 with a moderate nuclear cataract. An irregular ERM and slight PED were shown in optical coherence tomography (OCT). No obvious orange-red lesion was detected. The patient underwent vitrectomy + ERM peeling + cataract surgery. After the operation, large PED emerged, and indocyanine green angiography (ICGA) confirmed PCV. Four monthly injections of intravitreal ranibizumab were administered, but PED persisted. After focal laser therapy targeted to the polyps combined with ranibizumab treatment, PED was absorbed.

Conclusions: Careful evaluation for PCV before membrane peeling vitrectomy for ERM is important, as indolent PCV may be activated postoperatively. Anti-VEGF therapy accompanied by laser photocoagulation may be more effective for PCV polyps located away from the fovea.
\end{abstract}

Keywords: Polypoidal choroidal vasculopathy, Epiretinal membrane, Vitrectomy, Case report

\section{Background}

Epiretinal membrane (ERM) refers to the fibrocellular proliferation on the surface of the neurosensory retina [1], which mostly occurs in people aged over 50 , with a prevalence rate ranged from 2.2 to $9 \%$ among the different populations [2]. The majority of ERMs will remain stable and do not require therapy. The decision to

\footnotetext{
*Correspondence: chenyx@pumch.cn

'Yuelin Wang and Zhe Chen contributed equally to this work.

Weihong Yu and Youxin Chen are joint corresponding authors.

'Department of Ophthalmology, Peking Union Medical College Hospital,

Chinese Academy of Medical Sciences, 100730 Beijing, China

${ }^{2}$ Key Lab of Ocular Fundus Disease, Chinese Academy of Medical Sciences, 100730 Beijing, China
}

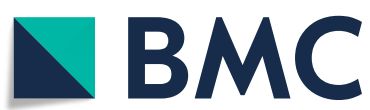

(c) The Author(s). 2021 Open Access This article is licensed under a Creative Commons Attribution 4.0 International License, which permits use, sharing, adaptation, distribution and reproduction in any medium or format, as long as you give appropriate credit to the original author(s) and the source, provide a link to the Creative Commons licence, and indicate if changes were made. The images or other third party material in this article are included in the article's Creative Commons licence, unless indicated otherwise in a credit line to the material. If material is not included in the article's Creative Commons licence and your intended use is not permitted by statutory regulation or exceeds the permitted use, you will need to obtain permission directly from the copyright holder. To view a copy of this licence, visit http://creativecommons.org/licenses/by/4.0/. The Creative Commons Public Domain Dedication waiver (http://creativecommons.org/publicdomain/zero/1.0/) applies to the data made available in this article, unless otherwise stated in a credit line to the data.

intervene usually depends on the severity of the patient's symptoms, such as how much they are bothered by their visual dysfunction. Early surgical intervention enables long-term visual recovery than delayed [3]. At present, vitrectomy combined with inner limiting membrane (ILM) peeling is considered to be an effective treatment option for ERM. By peeling the ERM and ILM, the traction of the macula can be relieved, the anatomic structure of the macula can be improved, which results in better visual function with a lower recurrence rate of ERM [4].

Regular ILM peeling vitrectomy seldom causes large pigment epithelial detachment (PED). We presented an unusual case of the activation of quiescent polypoidal 
choroid vasculopathy (PCV) after membrane peeling vitrectomy with ineffective monotherapy with anti-VEGF drugs. This report was organized in adherence to CARE guidelines (see Supplementary File 1) [5].

\section{Case presentation}

A 75-year-old female patient presented with metamorphopsia and decreased vision in her left eye for 2 years. She denied any history of diabetes or hypertension. Left eye examination: best-corrected visual acuity was 20/160, and slit-lamp examination revealed a moderate nuclear cataract. An irregular dense ERM (the "spaghetti sign") with macular edema and slight PED were detected on optical coherence tomography (OCT). No obvious orangered lesions or hemorrhages were seen. (see Fig. 1a and b).

The patient was diagnosed with ERM, wet age-related macular degeneration (wAMD), macular pucker, and age-related cataract in her left eye. The patient underwent phacoemulsification + pars plana vitrectomy + ILM peeling + intraocular lens implantation in the left eye, and the operation procedure was uneventful.

One month later, the visual acuity in her left eye was 20/80, and OCT showed significant PED with subretinal fluid (see Fig. 1c and d). Indocyanine green angiography (ICGA) showed a few polyps and adjacent large PED (see Fig. 1e) at the macula. PCV of the left eye was diagnosed afterward. After one injection of intravitreal ranibizumab, the height of the PED was decreased (see Fig. 1f), but after another three consecutive monthly intravitreal anti-VEGF drug (ranibizumab) injections, PED was not resolved (see Fig. 1 g). After laser photocoagulation combined with ranibizumab, the PED was absorbed (see Fig. $1 \mathrm{~h}$ ), and visual acuity improved to 20/63. The patient was satisfied with her present treatment.

\section{Discussion and conclusions}

PCV is a disease characterized by enlarged choroidal nodular lesions and abnormal branching of the choroidal vascular network, which is more common in Asians. Orange-red polyps are often detected, sometimes with subretinal hemorrhage, exudation, and PED [6]. However, some polyps may be ignored when PCV is indolent or combined with cataracts. According to the EVEREST criteria, focal hyperfluorescent lesions appearing before 6 min on ICGA is a necessary criterion for PCV diagnosis [7]. Therefore, careful inspection of the fundus with the ICGA exam was required.

The "spaghetti sign" refers to ILM dehiscence, which presents as hyporeflective linear spaces of varying lengths and depths interspersed with hyper-reflective bands, and protrusions of retinal nerve fiber layer (RNFL) tissue projecting into the posterior vitreous cavity on OCT $[8,9]$. The "spaghetti sign" sometimes appears because of posterior vitreous detachment, which creates a longitudinal pulling force on the ILM and RNFL but with residual tight adhesion. Vitrectomy and ILM peeling may pull the retina and cause tractional complications, such as PED or even retinoschisis. Therefore, the "spaghetti sign" may serve as a warning sign for membrane peeling vitrectomy.

But for this patient, PCV was quiescent preoperatively, vitrectomy had activated PCV with unknown mechanisms. We hypothesized that (1) intraocular pressure fluctuating during the operation could damage the choroidal vascular walls of polyps, thus disturbing the stability of the indolent PCV lesion; (2) after the vitreous was removed and the ILM was peeled, the restricting force on the retina was lost, which enabled the PCV lesions to inflate and activate; (3) during the membrane peeling vitrectomy operation process, iatrogenic strength may create an upward pulling force on the whole retina, especially with ERM (the "spaghetti sign"), which causes the junctional structure of the retinal pigment epithelium (RPE) and photoreceptor cells to loosen, and may lead to subretinal fluid effusion and large PED formation; (4) Vitrectomy can lead to a series of inflammatory responses, with the release of VEGF, PIGF, interleukin, and other inflammatory factors. Vascular permeability was changed, subretinal fluid was aggregated, and PED occurred. For our case, the presence and inactive nature of the PCV was not confirmed pre-operatively with ICGA, it is not known whether the subsequent surgery had any effect on the PCV. The development of actively leaking polyps may have simply been a coincidence rather than any direct effect of the surgery. Hence, ICGA should be used to evaluate PCV activity before membrane peeling vitrectomy.

For the treatment of PCV, anti-VEGF therapy is an important treatment choice. For this patient, using ranibizumab, a small-molecule antibody fragment, partially reduced her PED after the first injection, but after the second, third, and fourth monthly injections, PED persisted. At present, some researchers have reported the different effects of the fusion protein and small-molecule antibody fragment anti-VEGF drugs in the treatment of PED. Hata et al. conducted a retrospective study on 216 patients with AMD and found a greater reduction in PED in the aflibercept group than in the ranibizumab group [10]. It is worth of note that anti-VEGF agents approved in clinical practice, such as ranibizumab and aflibercept, are considerably different in terms of molecular interactions when they bind with VEGF [11]; therefore, characterization of such features can improve the design of novel biological drugs potentially useful in clinical practice. Other researchers suggest that [12] aflibercept reduced the thickness of the choriocapillaris in monkey eyes more significantly than ranibizumab, possibly because of an interaction between the Fc fragment and other molecules. Additional binding of aflibercept to 


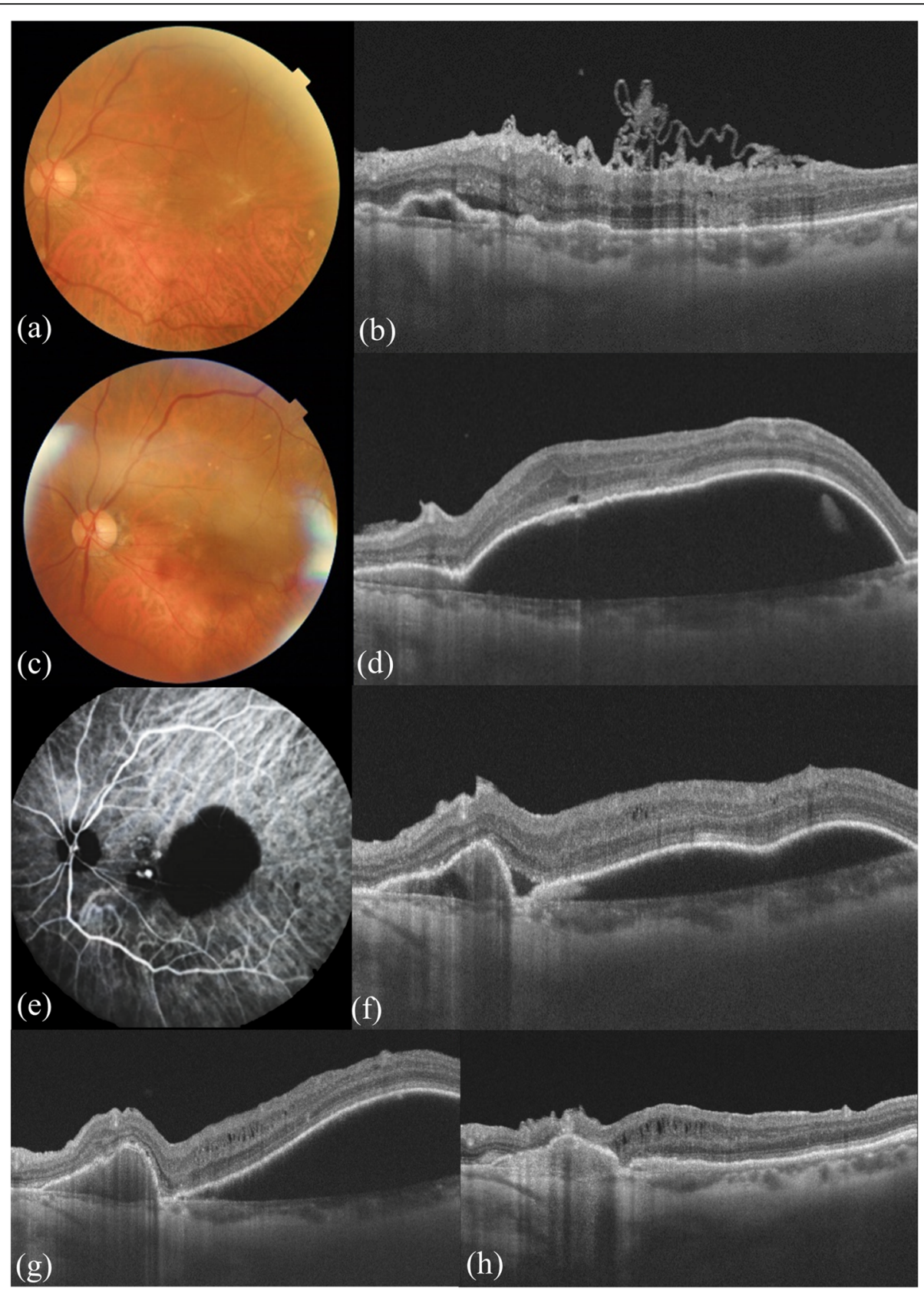

Fig. 1 a Fundus photograph showed the greyish-white ERM. b OCT (10 o'clock position) showed an irregularly folded epiretinal membrane (the "spaghetti sign") with edema. The nasal side of the macula showed a slight PED and a small amount of subretinal fluid. c,d One month after vitrectomy with ERM peeling, a significant increase in the height of PED was shown in the fundus and OCT, with orange polyps near the subtemporal vessels. e ICGA showed focal hyperfluorescent lesions at the nasal border of the PED lesion. $\mathbf{f}$ After one injection of intravitreal ranibizumab, the height of the PED was decreased. $\mathbf{g}$ After four monthly injections of intravitreal ranibizumab, PED persisted. $\mathbf{h}$ After focal laser therapy targeted to the polyps combined with ranibizumab, PED was absorbed

placental growth factor (PlGF) enabling higher binding affinity to VEGF than other VEGF inhibitors, and the development of autoantibodies before anti-VEGF therapy could also serve as potential explanations [13]. Therefore, fusion protein drugs can be a choice of alternatives for the treatment of refractory PED.
For this patient, considering that ranibizumab did not have an obvious therapeutic effect, laser photocoagulation was combined. The therapeutic target of laser photocoagulation is the position of high fluorescence on ICGA. After laser photocoagulation combined with ranibizumab, PED decreased significantly. For a long time, 
focal laser therapy has been used to ablate extrafoveal and extramacular polyps identified on ICGA. Focal laser therapy in combination with anti-VEGF therapy has been described to be effective in eyes with extrafoveal PCV. Lee et al. [14] reported stable vision or visual improvement in $75 \%$ of PCV patients, while Yuzawa et al. [15] demonstrated a decrease in visual acuity in $54 \%$ of PCV patients. The use of lasers may cause choroidal scars and subretinal or sub-RPE hemorrhage, which may influence vision. However, the combination of antiVEGF and laser therapy was reported to improve vision [16]. Compared with photodynamic therapy (PDT), laser photocoagulation is cheaper, more reachable, and has a similar therapeutic effect for PCV [17]. Therefore, laser therapy can also be effective for PCV treatment, especially with the combination of anti-VEGF drugs.

In conclusion, careful evaluation for PCV by ICGA before membrane peeling vitrectomy for ERM is important, as indolent PCV may be activated postoperatively. Fusion protein anti-VEGF drugs may be preferred for PED therapy, and anti-VEGF accompanied by laser photocoagulation may be effective for PCV polyps located away from the fovea.

\section{Abbreviations}

ERM: Epiretinal membrane; ICGA: Indocyanine green angiography;

ILM: Internal limiting membrane; OCT: Optical coherence tomography;

PED: Pigment epithelial detachment; PCV: Polypoidal choroidal vasculopathy: PDT: Photodynamic therapy; RNFL: Retinal nerve fiber layer; WAMD: Wet agerelated macular degeneration

\section{Supplementary Information}

The online version contains supplementary material available at https://doi. org/10.1186/s12886-021-02080-5.

\section{Additional file 1.}

\section{Acknowledgements}

YW would like to thank Di Cao for her support of the draft.

\section{Authors' contributions}

\#YW, ZC contributed equally to this paper. YW and ZC involved in the patient's diagnosis and treatment. WY contributed to manuscript revision. YC contributed to image evaluation and manuscript revision. All authors read and approved the final manuscript.

\section{Funding}

This work was supported by The Non-profit Central Research Institute Fund of the Chinese Academy of Medical Sciences (2018PT32029).

\section{Availability of data and materials}

The datasets used and/or analyzed during the current study are available from the corresponding author on reasonable request.

\section{Declarations}

\section{Ethics approval and consent to participate}

This study adhered to the tenets of the Declaration of Helsinki. No regular ethics approval is needed for case reports from the Peking Union Medical College Hospital Review Board. A copy of the written consent from the patient was obtained.

\section{Consent for publication}

Written informed consent was obtained from the patient for publication of this case report.

\section{Competing interests}

One of the authors, $Y C$, is a member of the editorial board of BMC

Ophthalmology. The other authors declare that they have no competing interests.

Received: 29 April 2021 Accepted: 24 August 2021

Published online: 06 September 2021

\section{References}

1. Steel DH, Lotery AJ. Idiopathic vitreomacular traction and macular hole: a comprehensive review of pathophysiology, diagnosis, and treatment. Eye (Lond). 2013;27(Suppl 1):1-21.

2. Mitchell P, Smith W, Chey T, Wang JJ, Chang A. Prevalence and Associations of Epiretinal Membranes. Ophthalmology. 1997;104(6):1033-40.

3. Flaxel CJ, Adelman RA, Bailey ST, Fawzi A, Lim JI, Vemulakonda GA, Ying GS. Idiopathic Epiretinal Membrane and Vitreomacular Traction Preferred Practice Pattern(R). Ophthalmology. 2020;127(2):145-83.

4. Fang $\mathrm{XL}$, Tong $\mathrm{Y}$, Zhou $\mathrm{YL}$, Zhao $\mathrm{PQ}$, Wang $Z \mathrm{ZY}$. Internal limiting membrane peeling or not: a systematic review and meta-analysis of idiopathic macular pucker surgery. Br J Ophthalmol. 2017;101(11):1535-41.

5. Gagnier JJ, Kienle G, Altman DG, Moher D, Sox H, Riley D, Group C. The CARE guidelines: consensus-based clinical case reporting guideline development. BMJ Case Rep. 2013;2013(1):1-4.

6. Cheung CMG, Lai TYY, Ruamviboonsuk P, Chen SJ, Chen Y, Freund KB, Gomi F, Koh AH, Lee WK, Wong TY. Polypoidal Choroidal Vasculopathy: Definition, Pathogenesis, Diagnosis, and Management. Ophthalmology. 2018;125(5):708-24.

7. Koh A, Lee WK, Chen LJ, Chen SJ, Hashad Y, Kim H, Lai TY, Pilz S, Ruamviboonsuk P, Tokaji E, et al. EVEREST study: efficacy and safety of verteporfin photodynamic therapy in combination with ranibizumab or alone versus ranibizumab monotherapy in patients with symptomatic macular polypoidal choroidal vasculopathy. Retina. 2012;32(8):1453-64.

8. Yeo JH, Kim JG. Bare retinal nerve fiber layer and internal limiting membrane tear associated with epiretinal membrane: OCT findings and visual outcomes. Graefes Arch Clin Exp Ophthalmol. 2020;258(7):1379-87.

9. Hussnain SA, Sharma T, Hood DC, Chang S. Schisis of the Retinal Nerve Fiber Layer in Epiretinal Membranes. Am J Ophthalmol. 2019;207:304-12.

10. Hata M, Oishi A, Tsujikawa A, Yamashiro K, Miyake M, Ooto S, Tamura H, Nakanishi $\mathrm{H}$, Takahashi A, Yoshikawa M, et al. Efficacy of intravitreal injection of aflibercept in neovascular age-related macular degeneration with or without choroidal vascular hyperpermeability. Invest Ophthalmol Vis Sci. 2014;55(12):7874-80.

11. Platania CB, Di Paola L, Leggio GM, Romano GL, Drago F, Salomone S, Bucolo C. Molecular features of interaction between VEGFA and antiangiogenic drugs used in retinal diseases: a computational approach. Front Pharmacol. 2015;10(6):1-13.

12. Julien S, Biesemeier A, Taubitz T, Schraermeyer U. Different effects of intravitreally injected ranibizumab and aflibercept on retinal and choroidal tissues of monkey eyes. Br J Ophthalmol. 2014;98(6):813-25.

13. Forooghian F, Chew EY, Meyerle CB, Cukras C, Wong WT. Investigation of the role of neutralizing antibodies against bevacizumab as mediators of tachyphylaxis. Acta Ophthalmol. 2011;89(2):e206-7.

14. Lee MW, Yeo I, Wong D, Ang CL. Argon laser photocoagulation for the treatment of polypoidal choroidal vasculopathy. Eye (Lond). 2009;23(1):145-8,

15. Yuzawa M. A Study of Laser Photocoagulation for Polypoidal Choroidal Vasculopathy. Jpn J Ophthalmol. 2003:47(4):379-84.

16. Gemmy Cheung CM, Yeo I, Li X, Mathur R, Lee SY, Chan CM, Wong D, Wong TY. Argon laser with and without anti-vascular endothelial growth factor therapy for extrafoveal polypoidal choroidal vasculopathy. Am J Ophthalmol. 2013;155(2):295-304. e291.

17. Nowak-Sliwinska P, van den Bergh H, Sickenberg M, Koh AH. Photodynamic therapy for polypoidal choroidal vasculopathy. Prog Retin Eye Res. 2013;37:182-99.

\section{Publisher's Note}

Springer Nature remains neutral with regard to jurisdictional claims in published maps and institutional affiliations. 